03

\title{
Диэлектрические потери и перенос заряда в легированном сурьмой монокристалле TIGaS $_{2}$
}

\author{
(C) С.М. Асадов ${ }^{1}$, С.Н. Мустафраева ${ }^{2,9}$ \\ ${ }^{1}$ Институт катализа и неорганической химии НАН Азербайджана, \\ Баку, Азербайджан \\ ${ }^{2}$ Институт ффизики НАН Азербайджана, \\ Баку, Азербайджан \\ ฯ E-mail: solmust@gmail.com
}

(Поступила в Редакцию 19 сентября 2017 г.

В окончательной редакции 25 сентября 2017 г.)

\begin{abstract}
Изучено влияние полуметаллической сурьмы $(0.5 \mathrm{~mol} \% \mathrm{Sb})$ на диэлектрические свойства и ас-проводимость выращенных методом Бриджмена-Стокбаргера монокристаллов на основе $\mathrm{TlGaS}_{2}$. Экспериментальные результаты по изучению частотной дисперсии диэлектрических коэффициентов и проводимости монокристаллов $\mathrm{TlGa}_{0.995} \mathrm{Sb}_{0.005} \mathrm{~S}_{2}$ позволили установить природу диэлектрических потерь, механизм переноса заряда и оценить параметры локализованных в запрещенной зоне состояний. Легирование монокристалла $\mathrm{TlGaS}_{2}$ сурьмой приводило к увеличению плотности состояний вблизи уровня Ферми и уменьшению среднего времени и расстояния прыжков.
\end{abstract}

Настоящая работа выполнена при финансовой поддержке Фонда развития науки при Президенте Азербайджанской Республики (проект 5.EIF-BGM-3-BRFTF-2+/2017).

DOI: $10.21883 /$ FTT.2018.03.45551.266

\section{1. Введение}

Кристаллы $\mathrm{TlGaS}_{2}$ относятся к тройным халькогенидным соединениям типа $\mathrm{TlGaX}_{2}$ (где $X-\mathrm{S}$, Se, $\mathrm{Te})$ со слоистой структурой. Квазидвумерность, широкозонность, структурная анизотропия, фазовые переходы, оптические и фотопроводящие свойства и другие особенности привлекают исследователей для лучшего понимания физики $\mathrm{TlGaS}_{2}$. Оптическая активность, высокая фоточувствительность в широком спектральном диапазоне $[1,2]$ отличают $\mathrm{TlGaS}_{2}$ от других полупроводников и делают его перспективным с практической точки зрения [3-6]. На основе $\mathrm{TlGaS}_{2}$ были предложены видимые и инфракрасные датчики света [7], а также высокочувствительные детекторы лазерного излучения [8-11]. Ha основе $\mathrm{TlGaS}_{2}$ можно получать материалы с непрерывно изменяющимися свойствами в зависимости от состава. Поэтому, а также ввиду возможных применений $\mathrm{TlGaS}_{2}$ в оптоэлектронных устройствах в видимом диапазоне в последнее время было уделено большое внимание изучению электрических, диэлектрических, фотовольтаических и фотоэлектрических $[12,13]$ свойств как чистого [14], так и легированного переходными и редкоземельными металлами соединения $\mathrm{TlGaS}_{2}[15-19]$. Было показано, что легирование кристаллов $\mathrm{TlGaS}_{2}$ приводит к существенному изменению диэлектрических коэффициентов монокристаллов и изменяет в них природу диэлектрических потерь.

Полуметаллическая сурьма $(\mathrm{Sb})$, как известно, является одной из основных примесей в полупроводниках. Однако при решении задачи, связанной с селективным легированием полупроводников сурьмой, сильная сегре- гация $\mathrm{Sb}$ осложняет получение материалов с воспроизводимыми характеристиками. Коэффициент сегрегации $\mathrm{Sb}$ например в кремнии, в интервале температур роста $573-823 \mathrm{~K}$ меняется почти на пять порядков.

Влияние легирования кристаллов $\mathrm{TlGaS}_{2}$ полуметаллическими примесями на их физические свойства мало изучено. Фотопроводимость монокристалла твердого раствора $\mathrm{TlGa}_{0.8} \mathrm{Sb}_{0.2} \mathrm{~S}_{2}$, выращенного методом Бриджмена-Стокбаргера, изучена в [20]. Спектр фотопроводимости монокристалла был измерен при $20 \mathrm{~K}$. В спектре наблюдались четыре пика при $504 \mathrm{~nm}$ $(2.460 \mathrm{eV}), \quad 525 \mathrm{~nm} \quad(2.361 \mathrm{eV}), \quad 571 \mathrm{~nm} \quad(2.171 \mathrm{eV})$ и $584 \mathrm{~nm}(2.123 \mathrm{eV})$. Указано, что пик при $504 \mathrm{~nm}$ соответствует запрещенной зоне. Пики при 571 и $584 \mathrm{~nm}$ могут быть отнесены к переходу электронов из валентной зоны на донорные уровни. В работе отсутствуют сравнительные данные с нелегированным $\mathrm{TlGaS}_{2}$ и не рассмотрено влияние сурьмы на физические свойства.

Учитывая вышеуказанное, в настоящей работе приведены результаты изучения влияния полуметаллической сурьмы $(0.5 \mathrm{~mol} . \% \mathrm{Sb})$ на диэлектрические свойства и электропроводность монокристаллов на основе $\mathrm{TlGaS}_{2}$, измеренные на переменном токе. Цель исследования состояла в том, чтобы выяснить природу диэлектрических потерь в $\mathrm{TlGa}_{0.995} \mathrm{Sb}_{0.005} \mathrm{~S}_{2}$ и механизм переноса заряда.

\section{2. Методика эксперимента}

Для решения вышеуказанной задачи использовали монокристаллические образцы $\mathrm{TlGaS}_{2}$ и $\mathrm{TlGa}_{0.995} \mathrm{Sb}_{0.005} \mathrm{~S}_{2}$. Оценка растворимости сурьмы в кристаллической решетке $\mathrm{TlGaS}_{2}$ с учетом эффективных ионных радиусов 
элементов указывает на то, что радиус $\mathrm{Sb}^{3+}(0.76 \AA)$ ближе к ионному радиусу $\mathrm{Ga}^{3+}(0.62 \AA)$, чем к радиусу $\mathrm{Tl}^{+}(1.5 \AA)$ [21]. Таким образом частичное замещение галлия сурьмой в кристаллах $\mathrm{TlGaS}_{2}$ соответствует условию образования твердого раствора замещения.

Исходными компонентами для получения образцов служили особо чистые химические элементы: Т1 (Тл00), $\mathrm{Ga}(\mathrm{Ga} 5 N)$, S (ос.ч. 165) и $\mathrm{Sb}$ (СУ0). Образцы синтезировали из взятых в стехиометрических соотношениях элементов путем непосредственного их сплавления в вакуумированных до $10^{-3} \mathrm{~Pa}$ кварцевых ампулах при $1000 \pm 3 \mathrm{~K}$ в течение 5-7 h. Образцы гомогенизировали при $750 \mathrm{~K}$ в течение $120 \mathrm{~h}$ и ампулы охлаждали до комнатной температуры в режиме выключенной печи. Завершенность синтеза кристаллов на основе $\mathrm{TlGaS}_{2}$ и их индивидуальность контролировали методом рентгеновского фазового анализа (РФА). РФА проводился на дифрактометре Bruker D8 ADVANCE с использованием $\mathrm{Cu} K_{\alpha}$-излучения при комнатной температуре.

Из синтезированных поликристаллов $\mathrm{TlGaS}_{2}$ и $\mathrm{TlGa}_{0.995} \mathrm{Sb}_{0.005} \mathrm{~S}_{2} \quad$ методом Бриджмена-Стокбаргера выращивали их монокристаллы $[14,16]$. Для этого поликристаллы измельчали и помещали в кварцевую ампулу длиной 8-10 cm с заостренным концом и внутренним диаметром $1 \mathrm{~cm}$. Вакуумированную до давления $10^{-3} \mathrm{~Pa}$ ампулу с поликристаллами помещали в двухтемпературную печь для выращивания монокристалла. В верхней зоне печи поддерживалась температура $1170 \pm 3 \mathrm{~K}$ (т.е. выше температуры плавления $\mathrm{TlGaS}_{2}$, которая составляет $1165 \mathrm{~K}$ ), а в нижней зоне $-1110 \pm 3 \mathrm{~K}$. Оптимальная скорость перемещения ампулы в печи равнялась $0.3-0.5 \mathrm{~cm} / \mathrm{h}$, а градиент температуры у фронта кристаллизации составлял $25 \pm 3 \mathrm{~K}$.

Монокристаллы $\mathrm{TlGaS}_{2}$, как известно, обладают сильной анизотропией физических характеристик и склонны к политипизму. Поэтому физические параметры этих кристаллов измеряли на образцах, взятых из одной технологической партии. Диэлектрические коэффициенты монокристаллов $\mathrm{TlGaS}_{2}$ и $\mathrm{TlGa}_{0.995} \mathrm{Sb}_{0.005} \mathrm{~S}_{2}$ измерены резонансным методом [22,23]. Диапазон частот переменного электрического поля составлял $5 \cdot 10^{4}-3.5 \cdot 10^{7} \mathrm{~Hz}$. Монокристаллические образцы из $\mathrm{TlGaS}_{2}$ и $\mathrm{TlGa}_{0.995} \mathrm{Sb}_{0.005} \mathrm{~S}_{2}$ для электрических измерений были изготовлены в виде плоских конденсаторов, плоскость которых была перпендикулярна кристаллографической $C$-оси образцов. В качестве электродов использована серебряная паста. Толщина образцов составляла $80-100 \mu \mathrm{m}$, а площадь обкладок $-5 \cdot 10^{-2} \mathrm{~cm}^{2}$.

Bce диэлектрические измерения проведены при $300 \mathrm{~K}$. Воспроизводимость положения резонанса составляла по емкости $\pm 0.2 \mathrm{pF}$, а по добротности $(Q=1 / \operatorname{tg} \delta) \pm 1.0-1.5$ деления шкалы. При этом наибольшие отклонения от средних значений составляли $3-4 \%$ для $\varepsilon^{\prime}$ и 7\% для $\operatorname{tg} \delta[22,23]$. Экспериментальное исследование образцов на основе $\mathrm{TlGaS}_{2}$ показало, что сегрегационное перераспределение $\mathrm{Sb}$ в выращенном монокристалле $\mathrm{TlGa}_{0.995} \mathrm{Sb}_{0.005} \mathrm{~S}_{2}$ не влияет на воспроизводимость физических свойств.

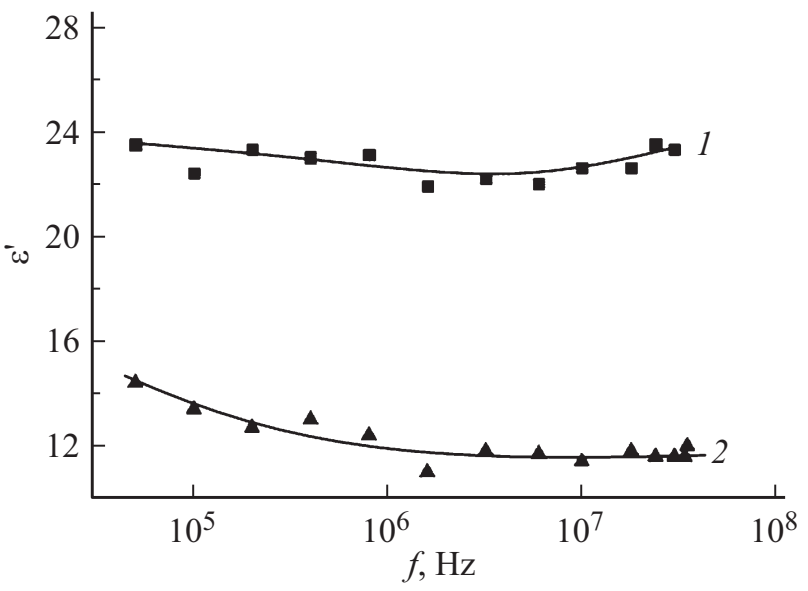

Рис. 1. Дисперсионные кривые $\varepsilon^{\prime}(f)$ для монокристаллов $\mathrm{TlGaS}_{2}$ (1) и $\mathrm{TlGa}_{0.995} \mathrm{Sb}_{0.005} \mathrm{~S}_{2}$ (2) при $300 \mathrm{~K}$.

\section{3. Результаты и их обсуждение}

Результаты РФА образцов на основе $\mathrm{TlGaS}_{2}$ показали, что параметры кристаллической решетки элементарной ячейки моноклинной сингонии (пр. гр. $C_{2 h}^{6}(C 2 / m)$ ) имели следующие значения: $a=10.299, b=10.284$, $c=15.175 \AA, \beta=99.603^{\circ}$. Эти характеристики согласуются с данными [24]. Образцы, в которых концентрация сурьмы $x=0.005$, также являются однофазными и при этом параметры решетки практически не меняются. На дифрактограмме легированного сурьмой образца $\mathrm{TlGa}_{0.995} \mathrm{Sb}_{0.005} \mathrm{~S}_{2}$ помимо дифракционных пиков фазы $\mathrm{TlGaS}_{2}$ не обнаружены дополнительные рентгеновские максимумы. Это указывает на то, что $\mathrm{Sb}$ изоморфно растворяется в $\mathrm{TlGaS}_{2}$.

На рис. 1 приведены частотные зависимости действительной составляющей комплексной диэлектрической проницаемости $\left(\varepsilon^{\prime}\right)$ образцов $\mathrm{TlGaS}_{2}$ и $\mathrm{TlGa}_{0.995} \mathrm{Sb}_{0.005} \mathrm{~S}_{2}$. Видно, что в $\mathrm{TlGaS}_{2}$ (кривая 1) во всем изученном диапазоне частот существенной дисперсии $\varepsilon^{\prime}$ не наблюдается. В $\mathrm{TlGa}_{0.995} \mathrm{Sb}_{0.005} \mathrm{~S}_{2}$ (кривая 2) с изменением частоты от $5 \cdot 10^{4}$ до $3.5 \cdot 10^{7} \mathrm{~Hz}$ значение $\varepsilon^{\prime}$ уменьшалось от 14.4 до 11.6. Наблюдаемое в экспериментах монотонное уменьшение диэлектрической проницаемости монокристалла $\mathrm{TlGa}_{0.995} \mathrm{Sb}_{0.005} \mathrm{~S}_{2}$ с ростом частоты от $5 \cdot 10^{4}$ до $3.5 \cdot 10^{7} \mathrm{~Hz}$ свидетельствует о релаксационной дисперсии [25]. Легирование кристаллов $\mathrm{TlGaS}_{2}$ сурьмой приводило к заметному уменьшению $\varepsilon^{\prime}$. Так, при $f=5 \cdot 10^{4} \mathrm{~Hz}$ значение $\varepsilon^{\prime} \mathrm{TlGa}_{0.995} \mathrm{Sb}_{0.005} \mathrm{~S}_{2}$ в 1.6 раза было меньше значения $\varepsilon^{\prime}$ в $\mathrm{TlGaS}_{2}$, а при $f=3.5 \cdot 10^{7} \mathrm{~Hz}$ - в 2 раза.

Частотные зависимости мнимой части комплексной диэлектрической проницаемости $\varepsilon^{\prime \prime}$ монокристаллов $\mathrm{TlGaS}_{2}$ (кривая 1) и $\mathrm{TlGa}_{0.995} \mathrm{Sb}_{0.005} \mathrm{~S}_{2}$ (кривая 2) приведены на рис. 2.

Значения тангенса угла диэлектрических потерь $(\operatorname{tg} \delta)$ в монокристаллах $\mathrm{TlGa}_{0.995} \mathrm{Sb}_{0.005} \mathrm{~S}_{2}$ существенно превышали значения $\operatorname{tg} \delta$ в $\mathrm{TlGaS}_{2}$ (рис. 3). Гиперболи- 


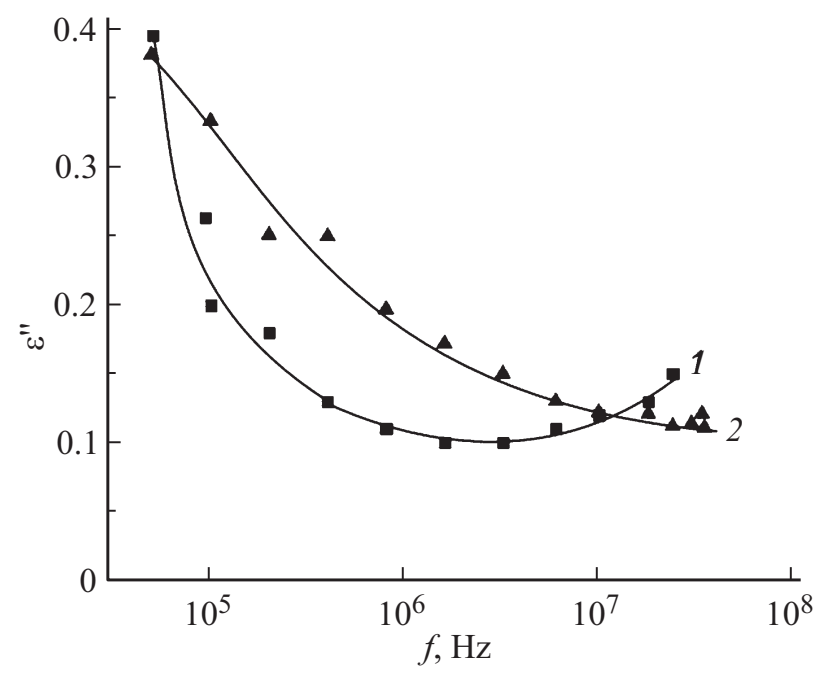

Рис. 2. Частотные зависимости мнимой составляющей комплексной диэлектрической проницаемости монокристаллов $\mathrm{TlGaS}_{2}$ (1) и $\mathrm{TlGa}_{0.995} \mathrm{Sb}_{0.005} \mathrm{~S}_{2}$ (2).

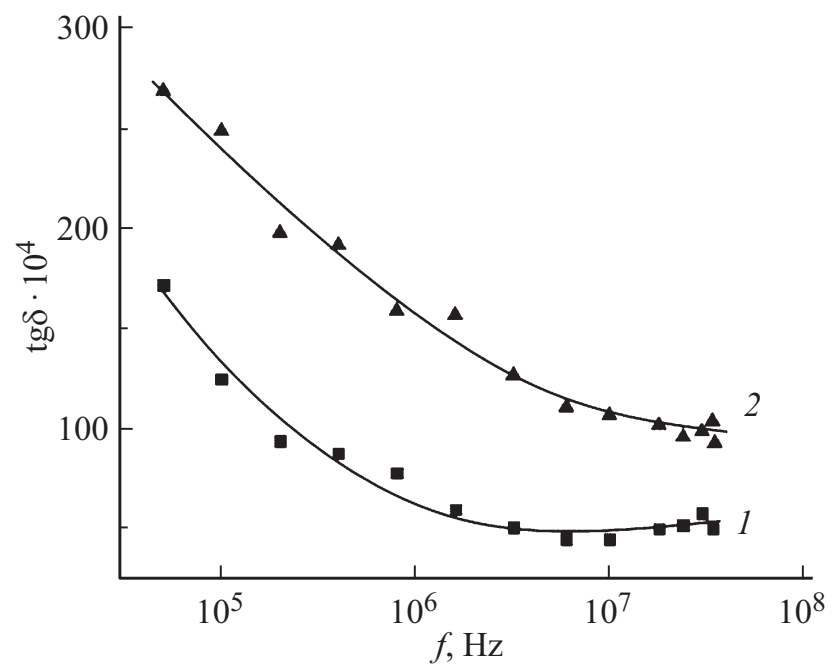

Pис. 3. Зависимости тангенса угла диэлектрических потерь $(\operatorname{tg} \delta)$ в монокристаллах $\mathrm{TlGaS}_{2}(1)$ и $\mathrm{TlGa}_{0.995} \mathrm{Sb}_{0.005} \mathrm{~S}_{2}(2)$ от частоты приложенного электрического поля.

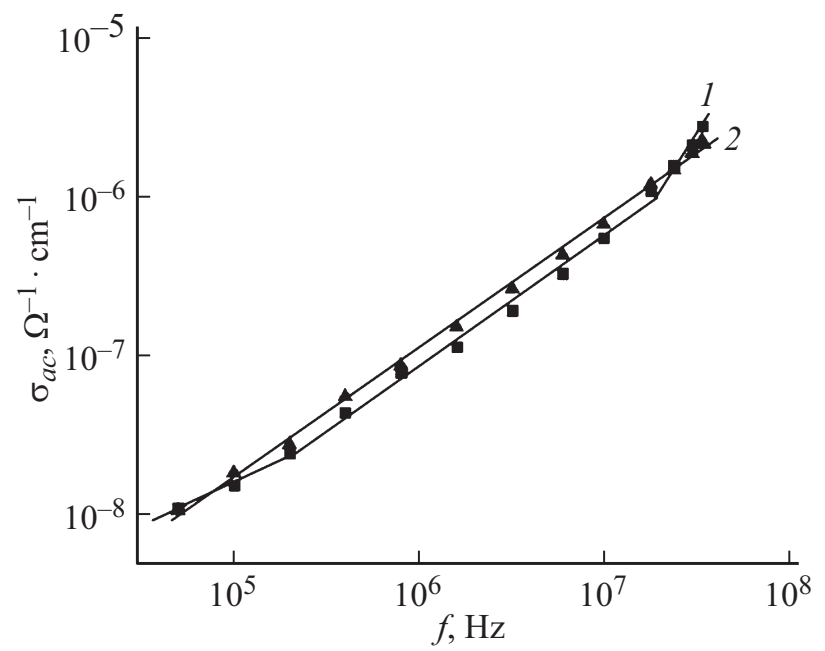

Рис. 4. Частотно-зависимая проводимость монокристаллов $\mathrm{TlGaS}_{2}(1)$ и $\mathrm{TlGa}_{0.995} \mathrm{Sb}_{0.005} \mathrm{~S}_{2}(2)$ при $T=300 \mathrm{~K}$. ческий спад $\operatorname{tg} \delta$ с увеличением частоты в изученных монокристаллах свидетельствует о потерях сквозной проводимости [25].

На рис. 4 представлены результаты изучения частотно-зависимой ас-проводимости монокристаллов $\mathrm{TlGaS}_{2}$ (кривая 1) и $\mathrm{TlGa}_{0.995} \mathrm{Sb}_{0.005} \mathrm{~S}_{2}$ (кривая 2) при $300 \mathrm{~K}$. В частотной области $5 \cdot 10^{4}-2 \cdot 10^{5} \mathrm{~Hz}$ acпроводимость монокристалла $\mathrm{TlGaS}_{2}$ изменялась по закону $\sigma_{\mathrm{ac}} \sim f^{0.6}$, а при $f=2 \cdot 10^{5}-2 \cdot 10^{7} \mathrm{~Hz} \sigma_{\mathrm{ac}} \sim f^{0.8}$. При $f>2 \cdot 10^{7} \mathrm{~Hz}$ имела место квадратичная зависимость $\sigma_{\mathrm{ac}} \sim f^{2}$. Дисперсионная кривая $\sigma_{\mathrm{ac}}(f)$ образца $\mathrm{TlGa}_{0.995} \mathrm{Sb}_{0.005} \mathrm{~S}_{2}$ во всей изученной области частот подчинялась закону $\sigma_{\text {ac }} \sim f^{0.8}$.

Ас-проводимость зонного типа, как известно, является в основном частотно-независимой вплоть до $10^{10}-10^{11} \mathrm{~Hz}$. Наблюдаемая нами экспериментальная зависимость $\sigma_{\mathrm{ac}} \sim f^{0.8}$ в кристаллах на основе $\mathrm{TlGaS}_{2}$ свидетельствует о том, что она обусловлена прыжками носителей заряда между локализованными в запрещенной зоне состояниями. Это могут быть локализованные вблизи краев разрешенных зон состояния или локализованные вблизи уровня Ферми состояния [26]. Но так как в экспериментальных условиях проводимость по состояниям вблизи уровня Ферми всегда доминирует над проводимостью по состояниям вблизи краев разрешенных зон, полученный нами закон $\sigma_{\mathrm{ac}} \sim f^{0.8}$ свидетельствует о прыжковом механизме переноса заряда по состояниям, локализованным в окрестности уровня Ферми. Предложенная в [27] формула для такой проводимости имеет вид

$$
\sigma_{\mathrm{ac}}(f)=\frac{\pi^{3}}{96} e^{2} k T N_{\mathrm{F}}^{2} a_{l}^{5} f\left[\ln \left(\frac{v_{\mathrm{ph}}}{f}\right)\right]^{4},
$$

где $e-$ заряд электрона; $k$ - постоянная Больцмана; $N_{\mathrm{F}}$ - плотность состояний вблизи уровня Ферми; $a_{l}=1 / \alpha-$ радиус локализации; $\alpha-$ постоянная спада волновой функции локализованного носителя заряда $\psi \sim e^{-\alpha r} ; v_{\mathrm{ph}}-$ фононная частота.

Согласно формуле (1) ас-проводимость зависит от частоты как $f\left[\ln \left(v_{\mathrm{ph}} / f\right)\right]^{4}$, т. е. при $f \ll v_{\mathrm{ph}} \sigma_{\mathrm{ac}}$ пропорциональна $f^{0.8}$. С помощью формулы (1) по экспериментально найденным значениям $\sigma_{\mathrm{ac}}(f)$ образцов $\mathrm{TlGaS}_{2}$ и $\mathrm{TlGa}_{0.995} \mathrm{Sb}_{0.005} \mathrm{~S}_{2}$ вычислили плотность состояний на уровне Ферми. Вычисленные значения $N_{\mathrm{F}}$ для $\mathrm{TlGaS}_{2}$ и $\mathrm{TlGa}_{0.995} \mathrm{Sb}_{0.005} \mathrm{~S}_{2}$ составляли $5.9 \cdot 10^{18}$ и $6.8 \cdot 10^{18} \mathrm{eV}^{-1} \cdot \mathrm{cm}^{-3}$ соответственно. Таким образом, легирование монокристалла $\mathrm{TlGaS}_{2}$ сурьмой приводило к увеличению плотности состояний вблизи уровня Ферми. При вычислениях $N_{\mathrm{F}}$ для радиуса локализации взято значение $a_{l}=14 \AA[14]$. Значение $v_{\mathrm{ph}}$ для $\mathrm{TlGaS}_{2}$ порядка $10^{12} \mathrm{~Hz}[28]$.

Согласно теории прыжковой проводимости на переменном токе среднее расстояние прыжков $(R)$ определяется по формуле [26]

$$
R=\frac{1}{2 \alpha} \ln \left(\frac{v_{\mathrm{ph}}}{f}\right) .
$$


В формуле (2) значение $f$ соответствует средней частоте, при которой наблюдается $f^{0.8}$-закон. Для монокристаллов $\mathrm{TlGaS}_{2}$ и $\mathrm{TlGa}_{0.995} \mathrm{Sb}_{0.005} \mathrm{~S}_{2}$ вычисленные по формуле (2) значения $R$ составляли 81 и $77 \AA$ соответственно. Эти значения $R$ примерно в 6 раз превышают среднее расстояние между центрами локализации носителей заряда в изученных монокристаллах. Значение $R$ позволило по формуле

$$
\tau^{-1}=v_{\mathrm{ph}} \exp (-2 \alpha R)
$$

определить среднее время прыжков в монокристаллах $\mathrm{TlGaS}_{2}$ и $\mathrm{TlGa}_{0.995} \mathrm{Sb}_{0.005} \mathrm{~S}_{2}: \tau=9.9 \cdot 10^{-8}$ и $4.4 \cdot 10^{-8} \mathrm{~s}$ соответственно.

По формуле

$$
\Delta E=\frac{3}{2 \pi R^{3} \cdot N_{\mathrm{F}}}
$$

в $\mathrm{TlGaS}_{2}$ и $\mathrm{TlGa}_{0.995} \mathrm{Sb}_{0.005} \mathrm{~S}_{2}$ оценен энергетический разброс локализованных вблизи уровня Ферми состояний: $\Delta E=150$ и $154 \mathrm{meV}$ соответственно. А по формуле

$$
N_{t}=N_{\mathrm{F}} \cdot \Delta E
$$

определена концентрация глубоких ловушек, ответственных за ас-проводимость в этих образцах: $N_{t}=8.8 \cdot 10^{17}$ и $10^{18} \mathrm{~cm}^{-3}$.

\section{4. Заключение}

В монокристаллах $\mathrm{TlGaS}_{2}$ и $\mathrm{TlGa}_{0.995} \mathrm{Sb}_{0.005} \mathrm{~S}_{2}$ со слоистой структурой получены воспроизводимые частотные зависимости тангенса угла диэлектрических потерь $(\operatorname{tg} \delta)$, действительной $\left(\varepsilon^{\prime}\right)$ и мнимой $\left(\varepsilon^{\prime \prime}\right)$ составляющих комплексной диэлектрической проницаемости и ас-проводимости $\left(\sigma_{\mathrm{ac}}\right)$ поперек слоев кристаллов в области частот $f=5 \cdot 10^{4}-3.5 \cdot 10^{7} \mathrm{~Hz}$. Легирование монокристаллов $\mathrm{TlGaS}_{2}$ сурьмой приводит к модифицированию дисперсионных кривых $\mathcal{\varepsilon}^{\prime}(f)$ и $\varepsilon^{\prime \prime}(f)$. Во всей изученной области частот в $\mathrm{TlGaS}_{2}$ и $\mathrm{TlGa}_{0.995} \mathrm{Sb}_{0.005} \mathrm{~S}_{2}$ имеют место потери на электропроводность. При высоких частотах ас-проводимость монокристаллов $\mathrm{TlGaS}_{2}$ и $\mathrm{TlGa}_{0.995} \mathrm{Sb}_{0.005} \mathrm{~S}_{2}$ подчинялась закономерности $\sigma_{\mathrm{ac}} \sim f^{0.8}$, характерной для прыжкового механизма переноса заряда по локализованным вблизи уровня Ферми состояниям. Оценены плотность и энергетический разброс состояний, лежащих в окрестности уровня Ферми, среднее время и расстояние прыжков в образцах $\mathrm{TlGaS}_{2}$ и $\mathrm{TlGa}_{0.995} \mathrm{Sb}_{0.005} \mathrm{~S}_{2}$. Сравнение показывает, что легирование монокристалла $\mathrm{TlGaS}_{2}$ сурьмой приводило к существенному изменению диэлектрических характеристик монокристаллов $\mathrm{TlGa}_{0.995} \mathrm{Sb}_{0.005} \mathrm{~S}_{2}$, увеличению плотности состояний вблизи уровня Ферми (от $5.9 \cdot 10^{18}$ до $6.8 \cdot 10^{18} \mathrm{eV}^{-1} \cdot \mathrm{cm}^{-3}$ ), уменьшению среднего времени (от $9.9 \cdot 10^{-8}$ до $4.4 \cdot 10^{-8} \mathrm{~s}$ ) и расстояния прыжков (от 81 до $77 \AA$ ). При этом концентрация глубоких ловушек, ответственных за аспроводимость в этих образцах, повышается от $8.8 \cdot 10^{17}$ до $10^{18} \mathrm{~cm}^{-3}$. Таким образом, установлено, что за счет легирования слоистого монокристалла $\mathrm{TlGaS}_{2}$ сурьмой можно управлять диэлектрическими коэффициентами и ас-проводимостью.

\section{Список литературы}

[1] I.G. Stamov, N.N. Syrbu, V.V. Ursaki, V.V. Zalamai. Optics. Commun. 298-299, 145 (2013).

[2] L. Nemerenco, N.N. Syrbu, V. Dorogan, N.P. Bejan, V.V. Zalamai. J. Luminesc. 172, 111 (2016).

[3] T. Kawabata, Y. Shim, K. Wakita, N. Mamedov. Thin Solid Films, 571, 589 (2014).

[4] B. Abay, H.S. Güder, H. Efeoğlu, Y.K. Yoğurtçu. Phys. Status Solidi B 227, 469 (2001).

[5] B. Gürbulak, S. Duman, A. Ateş. Czechoslov. J. Phys. 55, 93 (2005).

[6] Y. Shim, W. Okada, K. Wakita, N. Mamedov. J. Appl. Phys. 102, 083537 (2007).

[7] A.F. Qasrawi, N.M. Gasanly. Cryst. Res. Technol. 39, 439 (2004).

[8] A.F. Qasrawi, N.M. Gasanly. Phys. Status Solidi A 202, 13, 2501 (2005).

[9] I.M. Ashraf. J. Phys. Chem. B 108, 10765 (2004).

[10] A. Kato, M. Nishigaki, N. Mamedov, M. Yamazaki, S. Abdullayeva, E. Kerimova, H. Uchiki, S. Iida. J. Phys. Chem. Solids 64, 1713 (2003).

[11] A.A. Al Ghamdi, A.T. Nagat, F.S. Bahabri, R.H. Al Orainy, S.E. Al Garni. Appl. Surf. Sci. 257, 3205 (2011).

[12] C.-D. Kim, M.-S. Jin. New Physics: Sae Mulli 65, 11, 1068 (2015).

[13] M. Açıkgöz, P. Gnutek, C. Rudowicz. Solid State Commun. 150, 1077 (2010).

[14] С.Н. Мустафаева. ФТТ 46, 6, 979 (2004).

[15] С.Н. Мустафаева. Журн. радиоэлектроники 8, 1 (2008).

[16] С.Н. Мустафаева. Изв. РАН. Неорган. материалы 42, 5, 530 (2006).

[17] С.Н. Мустафаева. Журн. радиоэлектроники 4, 1 (2009).

[18] С.Н. Мустафаева, М.М. Асадов, Э.М. Керимова, Н.3. Гасанов. Неорган. материалы 49, 12, 1271 (2013).

[19] В.Г. Гуртовой, А.У. Шелег, С.Н. Мустафаева, Э.М. Керимова, С.Г. Джафарова. ФТТ 59, 8, 1479 (2017).

[20] M.-S. Jin, H.-J. Song. Current Applied Physics 3, 409 (2003).

[21] Дж. Хьюи. Неорганическая химия. Строение вещества и реакционная способность. / Под ред. Б.Д. Степина и Р.А. Лидина. Пер. с англ. Химия, М. (1987). 696 с.

[22] С.Н. Мустафаева, Д.М. Бабанлы, М.М. Асадов, Д.Б. Тагиев. ФТТ 57, 10, 1913 (2015).

[23] С.Н. Мустафаева. Все материалы. Энциклопедический справочник 10, 74 (2016).

[24] G.E. Delgado, A.J. Mora, F.V. Perezb, J. Gonzalez. Physica B 391, 385 (2007).

[25] В.В. Пасынков, В.С. Сорокин. Материалы электронной техники. 6-е изд. Лань, СПб.-М.-Краснодар. (2004). $368 \mathrm{c}$.

[26] Н. Мотт, Э. Дэвис. Электронные процессы в некристаллических веществах. Пер. с англ. Мир, М. (1974). 472 с.

[27] M. Pollak. Phil. Mag. 23, 519 (1971).

[28] К.Р. Аллахвердиев, Е.А. Виноградов, Р.Х. Нани. В кн.: Физические свойства сложных полупроводников. Элм, Баку (1982). С. 55. 